\title{
Lumen
}

Selected Proceedings from the Canadian Society for Eighteenth-Century Studies

\section{Pour une analyse informatisée du nom propre titulaire. L'exemple du roman français des Lumières}

\section{Elisabeth Zawisza}

Volume 16, 1997

Freedom and Boundaries

Émancipation et frontières

URI : https://id.erudit.org/iderudit/1012439ar

DOI : https://doi.org/10.7202/1012439ar

Aller au sommaire du numéro

Éditeur(s)

Canadian Society for Eighteenth-Century Studies / Société canadienne d'étude du dix-huitième siècle

ISSN

1209-3696 (imprimé)

1927-8284 (numérique)

Découvrir la revue

Citer cet article

Zawisza, E. (1997). Pour une analyse informatisée du nom propre titulaire.

L'exemple du roman français des Lumières. Lumen, 16, 53-70.

https://doi.org/10.7202/1012439ar 


\section{Pour une analyse informatisée du nom propre titulaire. L'exemple du roman français des Lumières}

Produit original de l'auteur autant que spécimen de l'art éditorial d'une époque, marque distinctive de l'oeuvre autant que carrefour de tendances et de modes, la page titulaire du roman ancien est particulièrement révélatrice de ce qui semble régir toute prise de parole, à savoir une tension irréductible entre liberté et restriction, entre l'émancipation tant convoitée par l'artiste et les limites socio-esthétiques qui entravent ses projets. Le choix du nom du fameux roman de Laclos illustre bien ce mouvement entre une stratégie de rituel et un besoin profond d'individualiser le texte. Au lieu commun d'intitulation qu'est devenu Le danger des liaisons, titre qui figure dans son manuscrit, Laclos préfère Les liaisons dangereuses - formule plus originale et intrigante, à l'instar du paratexte préfaciel et du contenu même de l'oeuvre. Dès la première page, ce roman se détache sur un fond d'une vingtaine d'ouvrages qui tiennent à avertir le public du Danger (ou des Dangers) «des passions», «des circonstances», «des préjugés», ou «d'une première faute» (cf. A. Martin et al, Bibliographie). Tout porte à croire, en effet, que le fait littéraire ne prend corps que dans une frange - parfois très imprécise, parfois rigoureusement définie par la loi - où le désir $\mathrm{d}$ 'indépendance créatrice affronte la volonté de l'Autre, les normes et les codes qui règlent l'émergence des textes. Et Michel Foucault de préciser:

Je suppose que dans toute société la production du discours est à la fois contrôlée, sélectionnée, organisée et redistribuée par un certain nombre de procédures qui ont pour rôle d'en conjurer les pouvoirs et les dangers, d'en maîtriser l'événement aléatoire, d'en esquiver la lourde, la redoutable matérialité. (L'ordre $d u$ discours 10-11)

Rien ne décrit mieux la condition difficile du roman des Lumières admirablement mise au jour par certaines stratégies du discours intitulant. Par la position particulière qu'elle occupe au seuil de l'oeuvre, la «marque du titre», signe par excellence, ${ }^{1}$ offre une voie d'accès incom- 
parable pour pénétrer dans cette écriture romanesque dont on sait qu'elle "dénote bien autre chose qu'un nom propre», rappelle G. Benrekassa, "captive et tributaire d'enjeux historiques avec lesquels elle entretient un rapport plus subtil et essentiel qu' on a voulu croire»(5).

Le festin nuptial dressé dans l'Arabie heureuse au mariage d'Esope, \& de Phedre E de Pilpaï avec trois autres fées, divisé en trois tables, Par M. de Palaidor..., Pirou, en Basse-Normandie, chez Florent A-Fable, à l'enseigne de la Vérité Dévoilée, 1700; Mylord Stanley, ou Le criminel vertueux, histoire tirée nouvellement des mémoires de l'illustre maison de $L^{*}$. $V^{*} \mathcal{E}$ mise en ordre par l'auteur d'An ${ }^{* *}$, Cadix, 1747, ou encore Le cadran des plaisirs de la cour ou les aventures du petit page Chérubin, pour servir de suite à la vie de Marie-Antoinette, ci-devant reine de France, Paris, chez les marchants de nouveautés, sans date; certes, ces «titres-sommaires» s'inscrivent en faux contre une sobriété tant recherchée par l'intitulation moderne. En optant pour des titres-idées ou des titres-énigmes, le romancier d'aujourd'hui ne craint pas d'inviter son lecteur à chercher des sens profonds dans la fiction devenue respectable. ${ }^{2}$ Or, mue par un contexte plus hostile au genre sans modèle et sans règles, la pratique titulaire $d^{\prime}$ autrefois se pose bon gré mal gré en publicité, en plaidoyer ou en manifeste. D'autres éléments titulaires ayant perdu leur force de persuasion directe, la titrologie moderne se concentre avant tout sur le titre proprement dit. A notre époque de référence, l'indication de l'auteur, le label de l'éditeur, voire même les vignettes et les épigraphes, qui confirment ou récusent le message titulaire, font partie intégrante de l'appareil d'intitulation. Faut-il s'étonner de voir que, au sein du discours d'escorte dont chaque élément se veut hautement fonctionnel, ce sont des noms propres qui se taillent la part du lion? Fictifs et authentiques, voilés et signalés, ces noms s'érigent d'abord en autant de signatures dans le sens derridien du terme, en autant de marques de présence (ou d'absence!) des personae engagées dans la «défense et illustration» du roman. Par ailleurs, leur prise de parole et leur silence s'inscrivent dans un moment crucial pour notre épistémè moderne, où l'autorité des universaux cède lentement la place à l'étude des cas particuliers, où une expérience singulière et constamment renouvelable commence à jouer un rôle primordial dans la saisie du réel. ${ }^{3}$ Rappelons, si besoin était, que la naissance de l'individu envisagé dans sa subjectivité est en même temps la condition sine qua non de l'émergence du roman avec ses formes et ses fonctions actuelles. Au sein du récit narratif, le nom propre est vite devenu l'outil le plus commode et le plus typique pour faire apparaître cet être singulier. ${ }^{4}$

Aborder, par le biais de son onomastique, la pratique titulaire des Lumières pour, en conclusion, dégager les particularités épistémiques et esthétiques de la production romanesque à l'étape charnière de son 
évolution, voilà un immense champ à survoler. ${ }^{5}$ En joignant dans une seule démarche les outils et les perspectives - complémentaires et divergents à la fois - de la linguistique et de l'analyse littéraire, notre projet $^{6}$ se propose d'étudier le statut et le fonctionnement du nom propre dans l'appareil titulaire des romans français publiés entre 1700 et 1800 . Centrée sur une pratique aussi multidimensionnelle, cette investigation se situe inéluctablement à l'intersection de plusieurs approches; elle met à contribution les acquis de l'onomastique et de la sémiotique, ${ }^{7}$ de $\mathrm{l}^{\prime}$ histoire et de la théorie littéraires, ${ }^{8}$ de la linguistique et du traitement informatique de données textuelles. ${ }^{9}$ A l'étape initiale de notre démarche, celle de la constitution du corpus des titres, ce sont les éditions originales qui retiennent notre attention; les rééditions et les traductions semblent poser d'autres problèmes méthodologiques. Il s'agit en fait de cerner ce geste d'intitulation originaire posé par les producteurs de textes romanesques, geste identique à celui des parents qui donnent un prénom à leur progéniture. ${ }^{10}$ Certes, tout comme le nom de baptême, le titre peut subir certaines modifications, voire même disparaître; ainsi L'ami de la fortune ou mémoires du marquis de S. $A^{* * *}$ de Maubert de Gouvest (Londres: Jean Nourse, 1754) se transforme dans une édition de 1761 en Mémoires historiques et politique du marquis de St. $A^{* * *}$. Mais l'on voit bien à quel point les deux dénominations et les motifs de leur changement sont révélateurs du statut du texte dénoté. Dans ce cas, il n'est plus question de transmettre l'information; ces termes génériques sur la page de titre veulent dire: "Veuillez considérer ce livre comme un roman», ou bien «prenez-le pour des mémoires». L'appareil titulaire saisi ici en plein exercice de sa fonction illocutoire (cf. G. Genette 15) montre le roman et son personnage, qui tantôt tiennent à s'intégrer dans une poétique de fiction, tantôt cherchent une filiation plus respectable avec un écrit authentique. A cet exemple puisé dans un monde littéraire fait pendant une décision «historique» de François-Marie Arouet de se muer en Voltaire, car ce nouveau Nom - apparemment propre et singulier, arbitraire et non productif l'aide en fait à se re-classer. En s'inscrivant en faux contre les théories logiques du nom propre vide de sens (S. Mill, A. Gardiner, J. Katz), notre projet trouve son fondement dans l'optique de ceux philosophes et sémioticiens (C. Lévi-Strauss, R. Barthes, F. Rigolot) pour qui le «nom de personne (dans le système linguistique, dans le roman), bien qu' «arbitraire», n'est cependant pas dépourvu de signification. Il ne se réduit pas à une pure étiquette; sa gratuité n'est qu'apparente, sa neutralité feinte...» (Ch. Grivel 129). Pour citer de nouveau Ch. Grivel:

Le nom est «parlant»; il énonce la personne qui le porte en lui assignant un rang, des qualités, en déclarant d'un point de vue ou d'un autre ses qualités (supposées ou non); il procède d'un choix, que marquent déjà les modes et les désaffectations 
successives dont il est l'objet. Le nom pare et se dépare; il circule dans les classes sociales, d'un niveau de roman à l'autre - et donc transmet du sens ... quelle que soit sa position (dans le texte ou non), le nom propre classe. ${ }^{11}$

Bref, notre analyse de l'onomastique titulaire veut mettre au jour des analogies sémantiques et fonctionnelles évidentes entre le nom propre, le titre, et l'oeuvre romanesque qui, tous les trois, dénotent leurs référents (individu, oeuvre, réalité extra-textuelle), tout en produisant des sens infiniment plus riches. Considérés dans leur fonction dénotative, le nom, le titre, et le roman identifient et signifient leurs objets, en visant ce fameux «effet de réel» théorisé par R. Barthes. Or, ces trois instances possèdent aussi des propriétés nécessaires pour produire d'autres effets; dans ce sens, «la signification du nom propre sera perçue comme un détour pour saisir l'idéologique du roman». ${ }^{12}$ Davantage une représentation du réel que son reflet, le roman, avec son titre et ses personnages, n'essaie-t-il pas, au fond, de «mettre le réel à bonne distance», voire même de «faire la concurrence au réel»? (G. Benrekassa 33-34). C'est donc pour mieux remplir la fonction poétique qui leur est inhérente que les récits fictifs mettent en place toute une série de dispositifs onomastiques: ils utilisent des connotations de noms réels, élaborées dans un contexte historique et social d'une époque; ils exploitent un réseau de signifiances littéraires déjà existant (comme dans L'anti-Therese, ou Juliette philosophe, nouvelle messine veritable, La Haye: 1750); ils forgent constamment de nouvelles relations intertextuelles. Or, et c'est déjà la question de méthode, ces particularités historiques d'une certaine pratique titulaire ne se laissent saisir que dans une confrontation $\mathrm{du}$ modèle général du titre avec un corpus de titres «homogène et soigneusement circonscrit». ${ }^{13}$ Notre corpus informatisé, sur lequel nous travaillons actuellement, offre au chercheur tous les avantages d'un tel ensemble fermé et homogène. Un rapide sondage portant sur trois tranches temporelles (1700-1709, 1750-1759, et 1790-1799) a confirmé que les pages titulaires foisonnent de noms propres. Indépendamment des fluctuations de cette production, environ $50 \%$ des titres contiennent un ou plusieurs noms propres désignant des êtres, des objets, des événements, et des lieux. Certains de ces noms renvoient au niveau diégétique (ND) du roman: ${ }^{14}$ ils désignent les acteurs (PR) de l'histoire («Amours d'Alzidor et de Charisée») ou servent d'opérateurs spatiaux (OS) («natif du village de Chezales») et temporels (OT) («les intrigues des règnes de Henry III et de Henry IV»). D'autres relèvent du rhématisme titulaire (NM); ils peuvent évoquer, par exemple, un des destinateurs (DR) du récit («tiré de l'ancienne chronique composée par Richard Coeur de Lion»), l'objet (OB) de la narration («Histoire politique et amoureuse du fameux cardinal Louis Portocarrero...»), ou encore son destinataire (DE) 
(«écrit et adressé par lui-même à son frère François»). Face à ce zèle des romanciers-onomaturges, la question qui se pose est celle de savoir comment circonscrire, comment faire parler un corpus si exubérant pour qu'il nous dévoile sa spécificité et ses modes de fonctionnement.

$C^{\prime}$ 'est ici que la perspective sémiotique s'avère particulièrement opérante, qui nous invite à envisager le phénomène $d^{\prime}$ 'intitulation sous quatre aspects: syntaxique, sémantique, sigmatique, et pragmatique. ${ }^{15} \mathrm{Et}$ nous voudrions inclure dans cet examen une étude bibliographique, $c^{\prime}$ est-à-dire une prospection des pages de titres des oeuvres particulières afin de dégager comment les structures matérielles du livre aident à mettre en relief le statut des noms. Étude d'autant plus importante que, comme le montre Hoek, elle est souvent négligée lors de la transcription bibliographique:

la bibliographie fait son travail de classification, en distinguant, parfois arbitrairement, un titre principal d'un titre secondaire, en apportant une ponctuation qui paraît appropriée et en négligeant, en bonne partie, la typographie $\mathrm{du}$ titre, la disposition des lignes, bref toute la mise en page. (Hoek 38)

A l'étape de l'analyse syntaxique des récurrences du corpus, phase qui tend à saisir la "grammaire titulaire» spécifique des romans du dix-huitième siècle, les derniers développements du traitement de données par ordinateur, les facilités de plus en plus grandes de repérer et de dénoter les éléments textuels, montrent une utilité incontestable des outils informatiques dans l'approche du fait littéraire. Dans notre travail d'étiquetage, il est possible aujourd'hui de dépasser le niveau strictement formel et lexical du vocable de l'appareil intitulant. Nous pouvons en effet tenter de cerner des relations entre le titre proprement dit $(T)$, l'indication de l'auteur (A) et l'adresse bibliographique (B); de saisir le nom propre dans ses relations fonctionnelles avec d'autres éléments du titre principal (TP) et des sous-titres (ST), ces parties constitutives de l'intitulation mises en rapport par des joncteurs (JC); bref, d'examiner les composantes relevées sur la page titulaire comme autant de cooccurrences. Une telle base de données informatisées, à haut niveau d'étiquetage et offrant des renseignements statistiques fiables, constitue un point de départ indispensable pour un examen détaillé d'autres aspects de l'intitulation retenus par les sémioticiens.

Un exemple permettra sûrement de mieux voir que ces niveaux $d$ 'investigation s'entrecoupent en fait dans l'analyse de tout appareil titulaire qui, par ailleurs, révèle constamment ses nombreuses relations avec un ensemble de discours intitulants. Ainsi, dans un roman anonyme Vénus à confesse ou lettres d'une comédienne retirée du spectacle à une de ses amies (En Phrigie, chez Esope, à l'enseigne de la vérité, 1751), c'est 
le nom d'une déesse romaine de l'Amour et de la Beauté qui est mis en relief par sa position hautement marquée dans le titre principal. L'étude de fréquence relèvera vite que ce type de patronymes - de consonance mythologique, «exotique» - sont plus fréquents dans la titrologie romanesque du dix-huitième siècle qu'on ne le pense. Alors que les chercheurs explorent surtout les techniques onomastiques de «vraisemblabilisation», celles qui ont participé à la naissance du roman réaliste, le corpus témoigne d'un intérêt persistant pour les possibilités connotatives des noms "non français", résidu d'une ancienne tradition de désigner les personnages fictifs. ${ }^{16}$ Mais notre travail d'étiquetage voudrait aller plus loin et capter les sens du prénom qui se dégagent de son rôle au sein de trois syntagmes nominaux formant l'appareil d'intitulation de l'ouvrage: le premier, qui relève du niveau diégétique du récit (Vénus à confesse), et deux autres qui signalent sa dimension métadiégétique (le sous-titre et l'adresse bibliographique). Sur leur fond, le nom inusité de Vénus - une métaphore qui, apparemment, ne se rapporte qu'au personnage principal - se doit en fait d'inciter le lecteur à prendre cette page de titre pour ce qu'elle est vraiment: un signe emblématique (une métaphore) des contradictions qui fondent la pratique romanesque et titulaire des Lumières. C'est que, d'une part, plusieurs éléments thématiques et rhématiques du titre («à confesse», «lettres», une «comédienne» qui s'érige en unique destinatrice de son discours en l'absence de l'auteur véritable) insistent sur le caractère authentique du texte et, ce faisant, essaient de jeter un voile sur sa provenance générique (IG). De l'autre, pourtant, en vue de promouvoir la valeur didactique d'un tel tableau de la vie, le label de l'éditeur - une de ces adresses fantaisistes dont notre corpus foisonne ${ }^{17}-n^{\prime}$ hésite pas à invoquer l'autorité d'un autre personnage antique, le fabuliste phrygien Esope.

En comparant l'impact didactique des lettres à celui, reconnu et apprécié, de l'apologue, l'adresse replace l'oeuvre dans un contexte fictif, en même temps qu'elle fait l'éloge des vertus curatives de la fiction contestée. Curieusement, cet exercice de promotion et de soutien ne tente aucunement de dissimuler son statut de lieu commun titulaire. Les «liseurs», qui disposaient du "code de la tradition intertextuelle» (L.H. Hoek), ont pu vite découvrir, avant même de les lire! la véritable nature des «lettres authentiques»: titre-affiche, titre-publicité n'agissent pas autrement. Le nom mythique de la déesse (déjà riche de sens) renvoyait le public averti aux pages titulaires des romans érotiques et galantes en vogue ${ }^{18}{ }^{1}$ 'actrice du sous-titre, elle, connotait le monde mal famé des déesses belles et frivoles du spectacle. Une formule thématique «retiré/e de» est également fort intéressante puisqu'elle aide à intégrer l'ouvrage dans le paradigme honnêteté/libertinage. Mise en place par Prévost 
dans Mémoires et aventures d'un homme de qualité qui s'est retiré du monde (1728-31), elle est reprise et revue, entre autres, par Lambert dans Mémoires et avantures d'une dame de qualité qui s'est retirée du monde (1739), et par Mouhy dans Mémoires d'une fille de qualité qui ne s'est point retirée $d u$ monde (1747), romans qui jouent sur des connotations morales de l'oeuvre de Prévost. Tout bien considéré, s'agit-il pour ce roman sur une comédienne de prôner des qualités moralisatrices du récit libertin? L'auteur qui préfère ne pas signer son oeuvre (TV) et l'éditeur (BE) qui, lui aussi, brille par son absence sur la première page, légitiment une telle lecture.

Or, en même temps, notre corpus permettra de reconstruire, avec tous les détails pittoresques et amusants, cette série d'instants où le «moi» cesse d'être «haïssable» et avoue son nom. "Signer une oeuvre de son vrai nom», dit Genette, «est un choix comme un autre et que rien n'autorise à juger insignifiant» (40). A l'époque où le romancier est traité d'«empoisonneur public, non pas des corps, mais des âmes des fidèles», le recours à l'anonymat, au pseudonymat, et à l'onymat est une décision lourde de sens. Qu'il était difficile autrefois d'exaucer le voeu de Rousseau: «Tout honnête homme doit avouer les livres qu'il publie». La case «Par» laissée vide et, ensuite, des signatures telles que «Par***», «Par Mr. C. de M.», «Par Messire Esprit, gentilhomme provençal, servant dans les troupes de Perse», «Par l'auteur des Mémoires de Versorand», «Par Mr. de Chansierge, ouvrage dédié au roi»: ces lieux communs de l'intitulation sont en fait aussi autant de réactions à l'anathème qui frappe le romancier. Et les éditeurs, les frères ennemis des écrivains, ne peuvent qu'agir en participants actifs de la querelle du roman. Les adresses bibliographiques où les noms vrais et fictifs, français et étrangers, se côtoient, où l'éditeur tait son nom, ou dans lesquelles un libraire réputé sert de prête-nom, renseignent admirablement sur le statut ambigu du genre romanesque. La fréquence de certaines formules marquent les hauts et les bas dans son irrésistible ascension. A cet égard, quoi de plus éloquent que ce quasi-degré zéro de l'intitulation: Néraïr et Melhoé, conte ou histoire, Ouvrage orné de digressions, Imprimé à ${ }^{* * *}$, se vend à ${ }^{* *}$, chez ${ }^{*}$, rue * à l'enseigne *. L'an de l'âge de l'auteur. C'est ainsi que, intégrés dans une adresse évocatrice et dans un titre principal aux sens si larges qu'on les précise dans un sous-titre particularisant, ${ }^{19}$ les anthroponymes-métaphores (Vénus, Esope) de notre exemple font voir une pratique scripturale qui tient autant à escamoter qu'à identifier et à désigner son objet. Ce geste de nommer si ambigu, car posé par des destinateurs exclus de leur discours intitulant, dévoile pourtant admirablement ce qui est au coeur de la pratique romanesque de cette période, à savoir les «interdits» qui frappent l'écriture réaliste et libertine, en obligeant ses producteurs 
à exploiter une formule pratique, mais déjà contestée par plusieurs: «ce n'est pas moi qui parle».

"Vénus» nous a fait parcourir un champ d'investigation très vaste, et pourtant l'exemple choisi est relativement simple du point de vue syntaxique. Le prénom (PR) peut s'accompagner d'un patronyme français (PF1) ou étranger (PF2) - ou d'un titre révélateur du statut et des origines du personnage (mademoiselle, milord, tsar - PT). L'adjonction des déterminants, dans les constructions telles que Le nouveau Faublas ou L'anti-Justine, présente encore un autre problème fascinant pour le littéraire et le linguiste. Marques les plus transparentes de l'intertextualité (nous y reviendrons), ces déterminants montrent un nom propre romanesque se transformer en nom commun. Une analyse détaillée de toutes ces occurrences, énoncée en termes statistiques, permettra de dégager les faits saillants de la nomination titulaire, mais aussi de relever des phénomènes onomastiques plus rares: vestiges de vieilles traditions ou signes avant-coureurs des changements qui se préparent. Si l'on songe maintenant à l'étape suivante, celle de l'étude sémantique, le nom de "Vénus» a fonctionné dans notre exemple comme une métaphore; or d'autres rôles sont encore plus fréquents dans les modes d'intitulation des Lumières. La critique ne cesse de souligner que l'emploi littéraire du nom reste toujours en rapport étroit avec son emploi social, que le nom (même s'il conteste le Code) est en fait, pour recourir au vocabulaire de Grivel, «l'usage d'un usage». Le nom titulaire de cette époque - dans sa fonction cardinale de contrat de lecture - identifie qui, comment, et dans quel but? Quels types de remotivations sont alors les plus fonctionnels? Plusieurs pratiques se laissent distinguer dans un va-et-vient entre ce qui, dans le nom propre, est singulier et individuel, et les possibilités qu'il offre de suggérer d'autre sens.

Ainsi, à la recherche de l'effet de véridiction, la dénomination fait semblant de se rapporter à un référent réel et précis, dénoté par son destin historique connu du lecteur; elle montre alors un créateur déguisé en chroniqueur ou en historien, qui se contente de transcrire des signes «naturels» parlant pour eux-mêmes. La pratique évolue cependant entre une Histoire des amours de Grégoire VII, du cardinal de Richelieu, de la princesse de Condé et de la marquise d'Urfé (1700) et Marie-Antoinette dans l'embarras ou correspondance de la Fayette avec le roi, la reine, la Tour-du-Pain $\mathcal{E}$ Saint-Priest (1790). Il convient d'étudier aussi comment l'indication de l'auteur et le label de l'éditeur, de même que le jeu de dédicaces et d'introductions étayent, ou mettent à nu, cette tactique de prise de parole. Car souvent le paratexte préfaciel est là pour corroborer le geste de l'auteur-historien. C. Freschot, par exemple, n'invente rien dans son Histoire anecdote de la cour de Rome; il se contente de faire parler «deux personnes mortes depuis peu, et qui ont bien été connues, l'une à la cour 
de Rome, et l'autre en celle de France» (Cologne, 1704, n.p.). D'autres noms, décidément plus nombreux à cette époque, montrent le romancier-onomaturge dans sa quête d'effet de vraisemblable. En imitant l'usage des prénoms, des patronymes, et des titres qui fonctionne dans la vie courante - or, Sophie et Jenny, Mr de Meilcour et Miss Belhove offrent des connotations de loin plus vastes que celles qu'implique un nom historique - , les écrivains réalisent un projet qui fonde l'écriture romanesque, celui d'attester «l'avènement de l'individu comme homme privé à valeur universelle», partant conférer à l'existence d'un être particulier un sens général qui la dépasse. ${ }^{20}$

C'est là aussi que semble résider l'intérêt de cette autre manière de nommer très populaire au dix-huitième siècle, exploitée avec un grand succès par Crébillon fils. Chez lui, les sigles, les initiales, et les astérisques (la Marquise de $\mathrm{M}^{* * *}$, le comte de $\mathrm{R}^{* *}[\mathrm{PV}]$ ) cachent des personnages présentés comme authentiques et uniques, tout en mettant en valeur «la rigueur et l'universalité de ses analyses psychologiques qui rivalisent avec les mathématiques» (L. Versini 178). Ce travail de remotivation, c'est-à-dire d'attribution du sens au nom arbitraire, est encore plus manifeste dans un recours à l'éponymie, qui permet d'établir un rapport direct, interne ou externe, entre le référé et le signifiant. Le premier cas, souvent évoqué, est celui de Voltaire qui, pour étayer sa vision du monde, invente ou utilise des formes dénominatives se référant directement aux caractéristiques du héros. Dans ses contes philosophiques, où les anthroponymes tels que Candide, l'Ingénu, ou Pococurante détruisent sciemment l'opacité naturelle du nom, l'écrivain exploite à fond les ressources sémantiques, graphiques, et phoniques de cette technique. ${ }^{21}$ L'éponymie externe, elle, fait parler le nom en réutilisant son sens étymologique, en rendant sa forme productive et fonctionnelle (Genette, Mimologiques 24 et passim). Voici un titre particulièrement éloquent, puisque tous ses éléments étayent l'argument posé déjà dans le prénom: Virginie ou la vierge chrétienne, histoire sicilienne pour servir de modèle aux filles qui aspirent à la perfection (1752). ${ }^{22}$ Cela dit, pour bien saisir les véritables fonctions des éponymes titulaires - comme c'est d'ailleurs le cas pour tout exercice d'onomastique littéraire - à l'examen du contenu sémantique du nom doit succéder son analyse sigmatique. Une association suggérée trouve-t-elle sa confirmation dans l'univers romanesque? S'agit-il, au contraire, d'une mystification? Question importante, car, pour citer la boutade de Hoek, dans la fiction «une Virginie n'est pas toujours vierge. . ., une Christine n'est pas toujours chrétienne» (Hoek 230). Si Jacques le Fataliste porte bien son surnom, Candide, lui, est érigé en témoin d'une mise à mort de l'optimisme.

Notre corpus contient aussi un nombre considérable de noms qui se proposent de véhiculer une extranéité plus au moins marquée - noms 
antiques, exotiques, fantaisistes, surnoms et sobriquets, souvent difficiles à classifier. Oeuvre du romancier qui n'occulte plus son travail d'onomaturge, cette poétique débridée peut chercher à insérer le texte dans la "germanicité» ou la «latinité», à suggérer une vague couleur locale, à forger dès le titre une image positive ou négative des acteurs de l'histoire. Ainsi, la lettre $\mathrm{Z}$ dans le prénom d'un personnage - pour sa rareté dans le code onomastique français - devient à un certain moment le signe par excellence de la thématique et de l'imaginaire orientaux (cf. M.-L. Dufresnoy 23). Pour savoir quelles connotations ont pu avoir pour les lecteurs des Lumières des noms tels que Alzidor et Pilpaï, Tibulle et Misapouf, Zeokinizul ou Ziri, il faudra tenter de reconstruire les codes ethnique, morphémique, culturel, et phonique à l'usage au dix-huitième siècle. Avec cette dénomination si créative, le romanesque se présente aux lecteurs dans son rôle fondamental de divertissement, et l'écriture dans sa fonction cardinale de jeu sur la langue, condition sine qua non pour que l'oeuvre littéraire puisse envisager d'autres objectifs. Car l'on sait qu'à cette époque d'ouverture et de mouvement, l'exotisme et l'altérité ne sont souvent que des masques qui cachent des messages jugés par certains subversifs. Dépourvus en principe de tout référent vrai ou vraisemblable, les anthroponymes si éloignés de la pratique sociale deviennent ainsi particulièrement utiles pour suggérer toutes sortes de sens: politique et pornographique, satirique et parodique. Dans cette catégorie de noms "hors du commun", les sobriquets et les surnoms (Royal Vilain, Javotte la Déhanchée, Jaco surnommé Henri Quatrième, ou Jacques le Fataliste) devront occuper une place à part, leur spécificité suscitant un intérêt particulier chez les linguistes et la critique littéraire. Contrairement aux noms fantaisistes, les surnoms, qui paraissent aujourd'hui si peu «réalistes», disposent paradoxalement d'un pouvoir accru de vraisemblabilisation. A l'époque où le surnom joue encore souvent le rôle d'un patronyme, ce type de nomination - qu'il s'agisse de désigner les caractéristiques morales et physiques du personnage, ou qu'il s'agisse d'évoquer sa vision du monde - peut surclasser, comme plus explicite et lisible, le nom propre en tant que producteur d'un effet de réel.

Comment, finalement, ne pas aborder le phénomène $d^{\prime}$ «intertextualité titulaire», geste particulièrement révélateur puisqu'il marie les effets du code linguistique aux ressources, riches et opérantes, de l'onomastique littéraire? Les Aventures et amours d'Ulisse avec la deesse Calypso. Histoire galante (1709), Naufrage des îles flottantes ou la Basiliade du célèbre Pilpaï, poème héroïque traduit de l'indien par Mr. $M^{* * * * * *}(1753)$, Galerie de tableaux ou contes nouveaux, Par un descendant de Boccace pour servir à l'éducation du beau sexe (1780), ou encore des intitulations déjà mentionnés, telles que Le nouveau Télémaque (1741) ou L'anti-Justine 
(1798) - les titres qui invoquent un livre, un auteur, un personnage ou un autre genre d'écriture constituent pour les lecteurs avertis autant de points de repère dans leur quête de signifiance. Cette pratique par excellence métadiscursive fait voir une oeuvre romanesque comme «une production en train de se faire, branchée sur d'autres textes, d'autres codes ... articulée de la sorte sur la société, l'Histoire non selon des voies déterministes mais citationnelles» (Barthes cité par Benrekassa 61 [nous soulignons]). En fait, avant même qu'une lecture attentive ne dégage l'ensemble de rapports que tisse l'oeuvre avec le «dehors» socio-culturel, l'intertextualité titulaire est là pour mettre au jour un incessant dialogue entre le roman et d'autres représentants de la République des Lettres: genres que le roman imite, dont il intègre les procédés, et genres qu'il dénigre, dont il réinterprète la forme et l'idéologie. ${ }^{23}$ Les noms puisés à pleines mains dans un réservoir des thèmes et des formes tantôt apportent à l'oeuvre la caution d'un autre texte, tantôt brossent un tableau du genre qui, sûr déjà de ses moyens, conteste haut et fort le discours de l'Autre.

Peut-on ne pas souscrire donc aux opinions de ceux qui insistent sur le rôle nodal, valorisé dès l'intitulation, du nom propre au sein du récit romanesque? Des travaux des formalistes russes au fameux mot de Barthes ${ }^{24}$ ce qu'on prenait longtemps pour un «désignateur rigide» se pose en "signe à part entière», un «élément central dans la sémiotique du personnage et dans la typologie narrative en général» (E. Nicole 233). Or, ce mot si essentiel - à côté des mots non essentiels, tels que les articles et les prépositions - émerge par un acte de baptême dans un emplacement qu'on appelait autrefois "des titres de frontispices», un support typographique qui ajoute un complément d'information aux éléments titulaires. Au dix-huitième siècle, en reconnaissant pleinement la fonction de séduction du paratexte, la «science de l'imprimerie» tente de préciser davantage les règles de construction de l'appareil intitulant. «Il est plus difficile qu'on ne se l'imagine, de bien faire une premiere page ... \& on peut même dire que c'est ici qu'on connaît la science d'un Compositaire», affirme le maître M.D. Fertel (79). Dans son ouvrage, qui foisonne d'exemples pratiques, il tient à conseiller ses confrères sur la façon d'arranger les composantes de l'intitulation pour qu'elles renforcent le message de l'auteur. Ainsi, le nom peut être mis en relief par la fréquence même avec laquelle il apparaîtra au seuil de l'oeuvre. Le modèle typographique du livre à l'usage au dix-huitième siècle permet de reprendre le titre et ses noms sous forme d'indication sommaire au dos de couverture, sur la page de titre, sur le frontispice (qui offre souvent en prime un portrait, muni d'inscription, du personnage principal), dans le faux titre, sur la première page du texte du roman, et même dans les titres courants en haut de page, où le nom répété maintes fois 
rappelle constamment l'identité du héros. Bien que son arsenal de moyens soit encore restreint, pour valoriser les noms qui figurent sur la page titulaire, l'imprimeur joue sur la disposition, la grandeur, et la grosseur des lettres, sur l'alternance des lignes pleines et courtes, sur le contraste entre les lettres romaines, italiques, et ornées, sur l'opposition des couleurs rouge et noir, ou encore il proportionne avec soin les blancs entre les lignes. Si, par contre, l'auteur de l'oeuvre relègue le nom propre au second plan, "dans des périodes qui suivent les mots essentiels», l'imprimeur se doit d'appuyer sa décision en faisant ces parties en cul de lampe ou en sommaire. ${ }^{25} \mathrm{Et}$ l'on sait que ces éléments du titre sont les premiers à disparaître, amputés par le travail des bibliographes et les exigences des éditions courantes.

Pour les dix-huitiémistes, la possibilité même d'aborder l'ensemble des discours intitulants des romans, de saisir les règles de la grammaire titulaire du genre dominant des Lumières, ouvre certainement un champs d'investigation littéraire et théorique fort intéressant. Considéré d'abord dans ses implications les plus vastes, ce corpus riche et hétérogène montre particulièrement bien l'urgence $d^{\prime} u n e$ onomastique titulaire conçue comme partie intégrante mais distincte de l'onomastique littéraire. Certes, à cette époque, les noms de titres participent de la même préoccupation de cerner l'individu que celle dont témoignent les énoncés romanesques, mais, apposées au seuil de l'oeuvre, ces signatures veulent attirer notre attention sur toutes les instancespersonae qui inventent, aident à transmettre et reçoivent le récit construit, lui aussi, autour des personnages; elles nous font voir une écriture qui réfléchit «à haute voix» sur les modes de sa production. Menée dans cette perspective, une analyse comparative du fonctionnement des noms propres dans le titre et à l'intérieur du monde fictif complétée par une étude complexe des rapports qu'entretiennent le discours liminaire, l'oeuvre et le public dans un contexte précis brossera un tableau à la fois des conditions d'écriture qui instituent l'individu comme objet et sujet de la création, et celui de l'existence humaine, tel que véhiculé par le romanesque des Lumières.

Par ailleurs, si l'on s'interroge sur l'utilité du corpus pour les études ponctuelles, on voit bien que tout titre - à l'instar du texte qu'il annonce - s'avère un mélange d'invention et de routine, dont l'originalité se dessine sur un fond des lieux communs implicites. Aussi faut-il, pour déterminer la spécificité du geste particulier de nommer (propre à un auteur, à une espèce romanesque ou à une étape dans l'évolution du genre) replacer ce geste dans l'ensemble de pratiques nominatives (sociales et littéraires) qui travaillent l'écriture d'une période. Point de repère indispensable pour diverses analyses des phénomènes d'intitu- 
lation, le corpus informatisé peut ainsi jeter les fondements d'une vaste étude d'ensemble portant sur l'onomastique titulaire dans les romans du dix-huitième siècle.

\section{ELISABETH ZAWISZA}

Queen's University

\section{Exemples de l'étiquetage du corpus des titres}

$<\mathrm{R} 1>$

$<\mathrm{A}><\mathrm{AF}>\mathrm{de}^{* *}<\mathrm{AT}>$ Mademoiselle $\left.</ \mathrm{AT}></ \mathrm{AF}></ \mathrm{A}\right\rangle$

$<\mathrm{T}\rangle<\mathrm{TP}\rangle\langle\mathrm{ND}\rangle\langle\mathrm{PR} 3>$ La nouvelle Talestris $</ \mathrm{PR} 3\rangle</ \mathrm{ND}\rangle</ \mathrm{TP}\rangle$ $<\mathrm{JC}>,</ \mathrm{JC}>$

$<\mathrm{ST}><\mathrm{NM}><\mathrm{IG}>$ histoire galante $</$ IG $></ \mathrm{NM}></ \mathrm{ST}>$ $<\mathrm{JC}>.</ \mathrm{JC}\rangle$

$<\mathrm{ST}><\mathrm{NM}\rangle<\mathrm{DR}>\mathrm{Par}<\mathrm{TT} 2>$ Mademoiselle $</ \mathrm{TT} 2\rangle\left\langle\mathrm{TV}>\mathrm{de}^{* *}</ \mathrm{TV}\right\rangle$ $</ \mathrm{DR}></ \mathrm{NM}></ \mathrm{ST}></ \mathrm{T}>$

$<$ B $><$ BLC $>$ Amsterdam $</$ BLC $>$

$<\mathrm{BE}>\mathrm{P}$. Marret $</ \mathrm{BE}>$

$<$ BDA $>1700</ \mathrm{BDA}></ \mathrm{B}>$

$<$ R6 $>$

$<\mathrm{A}\rangle\langle\mathrm{AF}\rangle$ [Bédacier, Catherine (Durand) $]</ \mathrm{AF}\rangle\langle/ \mathrm{A}\rangle$

$<\mathrm{T}><\mathrm{TP}\rangle<\mathrm{NM}\rangle<\mathrm{IG}>$ Les memoires secrets $</ \mathrm{IG}\rangle<\mathrm{OB}>$ de $<\mathrm{OS} 1>$ la cour de $<$ PR1 $>$ de Charles VII, $</$ PR1 $><$ PT2 $>$ roi de France $</$ PT2 $>$ $</ \mathrm{OS} 1></ \mathrm{OB}></ \mathrm{NM}></ \mathrm{TP}>$

$<\mathrm{JC}>.</ \mathrm{JC}\rangle$

$<\mathrm{ST}><\mathrm{NM}><\mathrm{DR}>$ Par $<\mathrm{TT} 2>$ Madame $\left.</ \mathrm{TT} 2><\mathrm{TV}>\mathrm{D}^{* * *}</ \mathrm{TV}\right\rangle$ $</ \mathrm{DR}></ \mathrm{NM}></ \mathrm{ST}></ \mathrm{T}>$

$<\mathrm{B}><$ BLA $>$ Paris $</$ BLA $>$

$<$ BE $>$ P. Ribou $</$ BE $>$

$<\mathrm{BDA}>1700</ \mathrm{BDA}></ \mathrm{B}>$

$<$ R7>

$<$ A $><$ AM $>$ [Bruslé de Montpleinchamp, Jean-Chrysostôme] $</$ AM $>$ $</ \mathrm{A}>$ 
$<\mathrm{T}><\mathrm{TP}><\mathrm{ND}><\mathrm{PQ} 4>$ Le festin nuptial dressé $<\mathrm{OS} 2>$ dans L'Arabie heureuse $</$ OS2 $>$ au mariage $<$ PR2 $>$ d'Esope $</$ PR2 $>$, $<$ PR2 $>\&$ de Phèdre $</ \mathrm{PR} 2><\mathrm{PR} 2>\&$ de Pilpai $</ \mathrm{PR} 2>$ avec $<\mathrm{PQ} 1>$ trois autres fées $</ \mathrm{PQ} 1></ \mathrm{PQ} 4></ \mathrm{ND}></ \mathrm{TP}>$ $<\mathrm{JC}>,</ \mathrm{JC}>$ $<\mathrm{ST}><\mathrm{NM}>$ divisé en trois tables $</ \mathrm{NM}></ \mathrm{ST}>$ $<\mathrm{JC}>$ : $</ \mathrm{JT}\rangle$ $<\mathrm{ST}><\mathrm{NM}><\mathrm{DR}>$ Par $<\mathrm{TT} 2>\mathrm{M} .</ \mathrm{TT} 2><\mathrm{TV}>$ Palaidor... $</ \mathrm{TV}>$ $</ \mathrm{DR}></ \mathrm{NM}></ \mathrm{ST}></ \mathrm{T}>$

$<\mathrm{B}><\mathrm{BLD}>$ Pirou, en Basse-Normandie $<$ /BLD $>$

$<\mathrm{BE}>$ chez Florent A-Fable, à l'enseigne de la Vérité Dévoilée $</ \mathrm{BE}>$ $<$ BDA $>1700</ \mathrm{BDA}></ \mathrm{B}>$

$<\mathrm{R} 9>$

$<$ A $><$ AM $>$ [Freschot, Casimir ] $</$ AM $></$ A $>$

$<\mathrm{T}\rangle\langle\mathrm{TP}\rangle\langle\mathrm{NM}\rangle<\mathrm{IG}>$ Relation historique $</ \mathrm{IG}\rangle\langle\mathrm{OB}\rangle$ de $<\mathrm{PQ} 4\rangle$ l'amour de $<$ PT2 $>$ l'empereur $<$ OS2 $>$ de Maroc $<$ /OS2 $><$ /PT2 $>$ $</ \mathrm{PQ} 4></ \mathrm{OB}></ \mathrm{NM}></ \mathrm{TP}>$

$<\mathrm{JC}>,</ \mathrm{JC}>$

$<\mathrm{ST}><\mathrm{NM}><\mathrm{DE}>$ pour $<\mathrm{PT} 2>$ Madame la princesse douarière [sic] $</$ PT2 $><$ PF1 $>$ de Conty $</$ PF1 $></$ DE $></$ NM $><$ ST $>$

$<\mathrm{JC}>,</ \mathrm{JC}>$

$<\mathrm{ST}><\mathrm{NM}><\mathrm{IG}>$ écrite en forme de lettres $</ \mathrm{IG}><\mathrm{DE}>$ à $<\mathrm{PQ} 1>$ une personne de qualité $</ \mathrm{PQ} 1></ \mathrm{DE}></ \mathrm{NM}></ \mathrm{ST}>$

$<\mathrm{JC}>$. $</ \mathrm{JC}>$

$<\mathrm{ST}><\mathrm{NM}><\mathrm{DR}>\mathrm{Par}<\mathrm{TT} 2>\mathrm{Mr}$. le comte $</ \mathrm{TT} 2><\mathrm{TV}>\mathrm{D}^{* * * *}</ \mathrm{TV}>$ $</ \mathrm{DR}></ \mathrm{NM}></ \mathrm{ST}></ \mathrm{T}>$

$<$ B $><$ BLC $>$ Cologne $</$ BLC $>$

$<$ BE $>$ P. Marteau $</$ BE $>$

$<\mathrm{BDA}>1700</ \mathrm{BDA}></ \mathrm{B}>$

\section{Notes}

1 Voir Hoek et $\mathrm{Cl}$. Duchet. C'est à la sociologie de la littérature et à la sémiotique que nous devons le regain d'intérêt actuel pour le paratexte; elles font voir l'intitulation comme une «zone indécise ... où se mêlent deux séries de codes: le code social, dans son aspect publicitaire, et les codes producteurs ou régulateurs du texte» (Duchet, cité dans Genette, Seuils 8).

2 C'est en pensant aux titres actuels que Grivel constate: «Le titre est flou. Sa brièveté fondamentale - tout titre fait figure de raccourci ou d'abrégé - oblige 
d'en opérer une lecture hésitante» (171). A preuve, le dernier roman de $\mathrm{N}$. Sarraute intitulé Ici! Et un rapide coup d'oeil sur un récent palmarès de $L^{\prime}$ Express révèle toujours la même prédilection des romanciers pour la structure à un terme (L'Alchimiste, Les Braban) et pour la structure binaire (Le Champ de personne, Mourir d'enfance), qui émergent seulement dans les années 30 du XIXe siècle (Duchet 60).

3 Pour une analyse des conditions épistémiques de la naissance du roman moderne, voir I. Watt et A. Renaut.

4 Selon la définition la plus commune et donc, nous le verrons, la plus problématique, du nom propre, celui-ci donne une identité à l'objet ou à la personne qu'il désigne; il en souligne le caractère unique et irremplaçable. Cf. E. Amon et Y. Bomati 144.

5 Duchet a déjà jugé utile d'analyser les noms propres «à part», avec tous leurs «éléments sémiques: féminité, exotisme, marques ethniques ou socio-hiérarchiques...» (63). Dans «A project for computer-assisted analysis of French prose fiction, 1751-1800", étude portant sur deux tranches temporelles (1751-1755 et 1771-1775), R. Frautschi offre un aperçu de la fréquence de certains prénoms et titres honorifiques. Martin, dans «Le roman de l'époque révolutionnaire», écarte les noms propres de son corpus, tout en insistant sur l'intérêt d'«une investigation plus approfondie du langage des titres» (94).

6 Ce projet, dont le présent article veut esquisser de grandes lignes, est codirigé par Elisabeth Zawisza et Greg Lessard et réalisé dans le cadre d'une recherche subventionnée par le Conseil de recherches en sciences humaines du Canada. Il porte sur un corpus informatisé de plus de 2000 titres constitué grâce à deux excellentes bibliographies, celle de S.P. Jones et celle de A. Martin, V.G. Mylne, R. Frautschi, qui, en principe, recensent la totalité de la production romanesque des Lumières. En ce qui concerne le formalisme d'étiquetage, nous tenons compte d'études récentes de Sperberg-McQueen et Burnard, dans lequel on propose un ensemble de normes SGML (Standard Generalized Markup Language) pour le traitement des textes informatisés. On trouvera un exemple de l'étiquetage du corpus des titres en fin d'article.

7 Cf., par exemple, Nicole; J. Bénard, M. Léonard, E. Nardout-Lafarge, Les noms $d u$ roman; Hamon.

8 Citons, relevant de ces approches, Versini, R. Démoris, et Genette, Mimologiques.

9 Voir Martin, «Laughter in the Old Regime», «Le roman européen au 18e siècle et la statistique bibliographique», et «French Fiction Titles in the Eighteenth Century».

10 Il s'agit bien d'une vision du nom (et du titre), selon laquelle un acte performatif du langage engendre un phénomène social: «Si le nom est l'apanage d'un être situé, son sens ne s'épuise pas là: il confère un titre qui fait de chacun un être irremplaçable ... Pour exister vraiment il faut d'abord être nommé. D'où l'importance du nom donné à l'enfant quand sa naissance est déclarée devant la société» (J. Goldstain 17).

11 Grivel 133. «Restent le nom de guerre, le pseudonyme littéraire ou artistique, résultats d'une tolérance, et qui sont, comme les sobriquets toujours en usage dans les campagnes, les derniers vestiges du temps où l'on se faisait vraiment un nom. Changer de nom, c'est faire peau neuve», conclut Markale (17; nous soulignons). 
12 F. Rigolot 12. Sur deux formes fondamentales de remotivation du Nom (culturelle et discursive) - car c'est d'elles qu'il est question ici - et sur les types de codes qui permettent au nom de connoter, voir Hoek 232-239.

13 Dans ce projet, nous nous servons du modèle général du titre, revu et développé pour le besoin de notre analyse, de Hoek, que ce sémioticien a élaboré dans sa Marque du titre.

14 Dans l'étiquetage des titres qui forment le corpus, ce niveau est marqué par les lettres ND. J'indiquerai désormais certaines de nos marques d'étiquetage à côté des éléments analysés, mais il est difficile, dans le cadre de cette présentation, de mentionner chacune d'environ 50 étiquettes dont nous nous servons.

15 L'étude syntaxique dégage les structures linguistiques du titre, ces composantes si caractéristiques de la production romanesque d'une période; la sémantique analyse le message véhiculé par les éléments diégétiques et métadiégétiques du titre; la sigmatique établit les rapports entre titres et leurs référés (le roman et d'autres titres et oeuvres). Cf. Hoek 36.

16 «. . avec Dupuis, Des Frans, chez Robert Challes, Valville, Habert, chez Marivaux, on est loin des héros précieux qui n'ont pas d'état civil, qui portent des noms antiques ou des noms de théâtre; on trouve encore, dans la première moitié du siècle, Cidalise et Clitandre, mais de plus en plus de tels noms se réfugient dans le conte» (Versini 177-178).

17 Voici quelques-unes de ces fausses adresses, éloquentes par ce qu'elles taisent: Pirou, en Basse-Normandie, chez Florent A-Fable, à l'enseigne de la Vérité Dévoilée, 1700; Gaznah, chez Fidele, imprimeur ordinaire de la Cour, l'an 1167 de l'Hégire et de l'ère chrétienne 1765; De l'imprimerie de la Liberté, sur la place de la Bastille, 1790. L'analyse de cette poétique, c'est encore un autre projet à entreprendre.

18 Vénus dans le cloître ou la religieuse en chemise, Vénus dans la vallée de Tempe, Vénus en rut ou la vie d une célèbre libertine, en voilà d'autres exemples tirés de notre corpus.

19 Précisons que, par rapport à l'étendue de l'information contenue dans le titre principal, le sous-titre peut viser une particularisation, une généralisation ou une identification (Hoek 201). Quel rôle joue le nom propre dans cette élaboration des sens?

20 Benrekassa 28. «En règle générale, le héros de roman est un idéologue», conclut Bakhtine (471).

21 Cf. E.T. Annandale. Et voici d'autres exemples trouvés sur les pages de titres des Lumières: Histoire de Camouflet, souverain potentat de l'empire d'Equivopolis (Equivopolis: 1751); M. de Fortengueule et M. de Palaidor (pour désigner les écrivains); Michel le Plagiaire et Jean Disant Vrai pour les éditeurs.

22 L'auteur du roman, qui s'appelle nomen omen le père Michel-Ange Marin, et dont le nom sur la page de titre a tout d'un procédé d'annomination, s'adonne à la production des romans d'inspiration morale et dévote: Agnes de Saint-Amour, ou la fervente novice (1750) ou Adélaïde de Witsbury, ou la pieuse pensionnaire. Avec la retraite spirituelle de huit jours (1750). Voir aussi un bon exemple de l'intérêt de ce type d'analyse dans T.-F. Belleguic.

23 Pour une analyse de la position spéciale du "genre en devenir» dans la hiérarchie des écrits, et sur sa nature dialogique, voir Bakhtine 442 et passim. 
24 «On peut dire que le propre du récit n'est pas l'action mais le personnage comme nom propre» (cité par Hoek 241).

25 Fertel 80 et passim. Son manuel formule aussi les règles de l'inscription sur la page de titre d'autres éléments hautement fonctionnels de l'appareil titulaire: «le nom des Auteurs» et leurs «qualités», de même que «les noms de Ville» avec «le nom, l'enseigne \& la demeure du Libraire» (82).

\section{Textes cités}

Amon, E., et Y. Bomati. Vocabulaire du commentaire du texte. Paris: Larousse, 1994.

Annandale, E. T. «Le nom propre dans les trois contes de Voltaire». Bénard et al. Les noms du roman. 33-41.

Bakhtine, M. «Récit épique et roman. Méthodologie de l'analyse de roman». Esthétique et théorie du roman. D. Olivier trans. Paris: Gallimard, 1978. 441-473.

Belleguic, T.-F. «Le Neveu de Rameau et les avatars d'un nom propre».

Bénard et al. Les noms du roman. 43-59.

Bénard, J., M. Léonard, E. Nadout-Lafarge, eds. Les noms du roman. Montréal: Paragraphe, Département d'Études françaises, Université de Montréal, 1994.

Benrekassa, G. Fables de la personne. Pour une histoire de la subjectivité. Paris: PUF, 1985.

Démoris, R. «De l'usage du nom propre: le roman historique au XVIIIe siècle». Revue d'histoire littéraire de la France 2-3 (1975): 268-288.

Duchet, $\mathrm{Cl}$. «La fille abandonée et La Bête humaine, éléments de titrologie romanesque». Littérature 12 (1973). 49-73.

Dufresnoy, M.-L. L'Orient romanesque en France 1704-1789. Montréal: Editions Beauchemin, 1974.

Fertel, M. D. La science pratique de l'imprimerie contenant des instructions tres-faciles pour se perfectionner dans cet art. Saint Omer: M. D. Fertel, 1723.

M. Foucault. L'ordre du discours. Paris: Gallimard, 1971.

Frautschi, R. «A project for computer-assisted analysis of French prose fiction, 17511800». A.J. Aithen, R. W. Bailey, eds. The Computer and Literary Studies. Edinburg: N. Hamilton-Smith, 1973. 183-195.

Genette, G. Mimologiques, Voyages en Cratylie. Paris: Seuil, 1976. Seuils. Paris: Seuil, 1987.

Goldstain, J. «Le nom dans le Nouveau Testament». Corps écrit 8 (1983): 17-27.

Grivel, Ch. Production de l'interêt romanesque. Une état du texte (1870-1880), un essai de constitution de sa théorie. Paris: Mouton, 1973.

Hamon, Ph. «Pour le statut sémiologique du personnage». Poétique du récit. Paris: Seuil, 1977. 42-61.

Hoek, L. H. La marque du titre. Dispositifs sémiotiques d'une pratique textuelle La Haye: Mouton, 1981. 
Jones, S. P. A List of French Prose Fiction. New York: Wilson, 1939.

Markale, J. «Le nom, la parole et la magic». Corps écrit 8 (1987): 29-39.

Martin, Angus. «French Fiction Titles in the Eighteenth Century: A Computer-Assisted Analysis». Studies in Voltaire and the Eighteenth Century 304 (1992): 994-998.

«Laughter in the Old Regime: a Computer Analysis of Joke Titles in Seventeenthand Eighteenth-Century France». Australian Journal of French Studies 36.3 (1989): 230-241.

. «Le roman de l'époque révolutionnaire (1789-1794): conclusions tirées du traitement informatique des titres». Lendemains 55-56 (1989): 87-95

. «Le roman européen au 18e siècle et la statistique bibliographique». Dix-huitième siècle 25 (1993): 101-114.

Martin, A., V.G. Mylne, R. Frautschi, eds. Bibliographie du genre romanesque français 17511800. London: Mansell; Paris: France Expansion, 1977.

Nicole, E. «L'onomastique littéraire». Poétique 54 (1983): 233-253.

Sperberg-McQueen, C. M., et L. Burnard, eds. Text Encoding Initiative: Guidelines for Electronic Text Encoding and Interchange (TEI P3). Chicago et Oxford: http://www.uic.edu/orgs/tei/p3/p3x/p3x.html (16 mai 1997).

Renaut, A. L'ère de l'individu. Paris: Gallimard, 1989.

Rigolot, F. Poétique et onomastique. L'exemple de la Renaissance. Genève: Droz, 1977.

Versini, L. «De quelques noms de personnages dans le roman du XVIIIe siècle». Revue d'histoire littéraire de la France 2 (1961): 177-187.

Watt, Ian. «Réalisme et forme romanesque». Littérature et réalité. Paris: Seuil, 1982. 11-46. 